\title{
VARIAÇÃO DAS PERDAS MAGNÉTICAS DURANTE EN VELHECIMENTO DE UM AÇO ELÉTRICO
}

Kleiner Marques Marra 1 Vicente Tadeu Buono 2

\section{Resumo}

Submeteu-se um aço elétrico semiprocessado com teor residual de carbono de 30 ppm e com adições de Al e $\mathrm{Si}$, a diferentes tempos de encharque numa temperatura de $210^{\circ} \mathrm{C}$. Foi verificado que a perda magnética histerética aumentou continuamente até $24 \mathrm{~h}$, tendendo a se manter invariável para mais longas exposições. As perdas parasítica e anômala se mostraram insensíveis ao tratamento de envelhecimento. Foi constatado, por atrito interno e microscopia eletrônica de transmissão, que a precipitação de carbono foi responsável pelo fenômeno do envelhecimento. Por microscopia eletrônica de varredura, ficou evidenciado que a evolução do envelhecimento foi acompanhada pelo crescimento da quantidade de carbonetos maiores que $0,1 \mu \mathrm{m}$.

Palavras-chave: Aço elétrico; Perda magnética; Envelhecimento magnético.

\section{VARIATION OF MAGNETIC LOSSES DURING AGING OF AN ELECTRICAL STEEL}

\begin{abstract}
An ultra-low carbon steel (30incts soaking times at $210^{\circ} \mathrm{C}$. The core loss increased continuously until around 24 h. After such time little modifications were verified. It was found that only the hysteresis loss component changed during the aging treatment. By internal friction and transmission electron microscopy it was seen that carbon precipitation caused the magnetic aging. By scanning electron microscopy it could be concluded that the increase of aging index originated from the enlarging of the number of carbides bigger than $0,1 \mu \mathrm{m}$.
\end{abstract}

Key words: Electrical steel; Core loss; Magnetic aging.

\section{INTRODUÇÃO}

Aços elétricos semiprocessados são materiais aplicados especialmente em núcleos de motores e pequenos transformadores. Para 0 desenvolvimento de boas propriedades magnéticas, esses aços são submetidos usualmente a tratamentos térmicos, nos consumidores, que visam, além de reduzir o teor de carbono, promover crescimento de grão, formar uma textura cristalográfica mais adequada e desenvolver isolamento elétrico pela criação de uma camada contínua de óxidos sobre a superfície do aço.

A redução do teor de carbono do aço no tratamento térmico de descarbonetação é muito importante para a melhoria das propriedades magnéticas pois promove queda na perda no núcleo e, concomitantemente, eleva a permeabilidade, além de influenciar a susceptibilidade ao envelhecimento magnético.

0 envelhecimento tem origem nas partículas finas de segunda fase presentes na matriz dos aços. Estas partículas são prejudiciais ao processo de magnetização por funcionar como obstáculos à movimentação dos domínios magnéticos, elevando a dissipação da energia magnética.(1,2) Essa energia dissipada, ou perda magnética no núcleo, pode ser decomposta em três parcelas: a perda histerética $P_{h}$ (relativa à dissipação de energia em função da movimentação dos domínios magnéticos durante a aplicação do campo magnético de excitação), a perda parasítica $P_{p}$ (relativa à dissipação de energia devido a geração das correntes elétricas parasíticas na matriz metálica) e a perda anômala $P_{a}$ que é igual à diferença entre a perda total e a soma das outras duas parcelas. A componente histerética é bastante sensível à presença de partículas de segunda fase, principalmente na faixa de diâmetro de 0,1 a $1,0 \mu \mathrm{m}$.(3)

Se, durante a magnetização, o aquecimento, resultante da conversão de parte da energia magnética em energia térmica, for capaz de causar a precipitação de novas partículas ou coalescer as partículas já existentes, a perda magnética do aço cresce e a permeabilidade decresce, implicando no aparecimento do envelhecimento magnético. $\mathrm{N}$ ormalmente, a temperatura atingida no núcleo de transformadores e motores, durante sua operação, não é muito alta, ficando geralmente abaixo de $200^{\circ} \mathrm{C}$.

\footnotetext{
1 Engenheiro M etalurgista, Dr., Superintendência do Centro de Pesquisa e Desenvolvimento da Usiminas; Ipatinga, M G.

2 Físico, Dr., Professor Adjunto do Curso de Engenharia Metalúrgica e de Materiais da UFM G; Belo H orizonte, MG.
} 
Deve ser ressaltado que a baixa susceptibilidade ao envelhecimento é um requisito importante para aços destinados a aplicações magnéticas. 0 envelhecimento magnético é normalmente estudado em laboratório pelo aumento da perda magnética após um certo tempo de exposição do aço a uma determinada temperatura de encharque. Para isto, usa-se 0 índice de envelhecimento (IE), que é a variação relativa da perda magnética provocada pelo envelhecimento, ou seja:

$\mathrm{IE}(\%)=\frac{\mathrm{P}_{\mathrm{aw}}-\mathrm{P}_{\text {mata }}}{\mathrm{P}_{\text {awa }}} 100$

Onde:

$\mathrm{P}_{\text {antes }}=$ perda magnética antes do tratamento de envelhecimento,

$P_{\text {env }}=$ perda magnética após 0 tratamento de envelhecimento.

$\mathrm{N}$ este trabalho foi adotado o valor de $5 \%$ para IE como 0

limiar do envelhecimento. Foi empregado um aço elétrico extrabaixo carbono submetido a um processo de envelhecimento pela aplicação de tratamentos térmicos compostos de encharque à $210^{\circ} \mathrm{C}$ e tempos de até $600 \mathrm{~h}$.

\section{PROCEDIMENTO EX PERIMENTAL}

Foi utilizado um aço semiprocessado com teor inicial de carbono de 66 ppm e teores de silício e de alumínio de, respectivamente, $0,60 \%$ e $0,20 \%$. Esse material, na espessura de $0,50 \mathrm{~mm}$, foi descarbonetado na forma de lamelas por duas horas, em um forno piloto do tipo mufla, sob temperatura de $760^{\circ} \mathrm{C}$. Foi empregada uma atmosfera do tipo $\mathrm{HN}(90 \%$ de nitrogênio e $10 \%$ de hidrogênio), com diferentes graus de umidificação, de modo a serem obtidos teores residuais de carbono variando de 10 até $66 \mathrm{ppm}$.

0 teor de carbono do aço, antes e após a descarbonetação, foi medido em analisador Leco, modelo 444LS, sendo dado como média de seis resultados individuais.

A perda magnética no núcleo (perda total) foi medida num equipamento do tipo single tester, modelo BDS280, fornecido pela Brockhauss, em lamelas do aço ( $280 \mathrm{~mm} \mathrm{X} 30 \mathrm{~mm} \mathrm{X}$ espessura). Foi usada uma condição de magnetização com indução de 1,0 T, obtido com corrente indutora de freqüência alternada $(60 \mathrm{~Hz})$. Cada valor de perda foi tomado como a média aritmética de três medições individuais. Os resultados indicaram que houve uma nítida tendência de decréscimo da perda com a redução do teor de carbono.

A pós a submissão das amostras descarbonetadas a um ciclo de tratamento de envelhecimento a $210^{\circ} \mathrm{C}$, por $24 \mathrm{~h}$, observouse que o máximo valor de perda (maior IE) ocorreu para os teores de carbono residual de 25 a $40 \mathrm{ppm}$, figura 1. Por outro lado, abaixo de 20 ppm não houve envelhecimento $(I E<5 \%)$.

Com isto, optou-se por investigar 0 envelhecimento magnético em amostras com 30 ppm de carbono residual. Assim, para efeito de se estudar a evolução deste fenômeno, foram aplicados tratamentos a $210^{\circ} \mathrm{C}$, sob tempos variados de encharque (4 h, 8 h, 24 h, 48 h, 100 h, 200 h, 400 h e 600 h).

As perdas magnéticas das amostras envelhecidas foram separadas nas componentes parasíticas, histeréticas e anômalas pelo método de Bertoti.(4)
A evolução do envelhecimento magnético foi avaliada também pela identificação e quantificação das partículas de segunda fase na matriz metálica em microscópio eletrônico de varredura (MEV) e microscópio eletrônico de transmissão (MET).

No caso do MEV, usaram-se imagens geradas num equipamento da Cambridge (modelo Stereoscan 360) que, posteriormente, foram transferidas para um analisador de imagens (Q uantimet 600HR, da Leica). 0 software disponível no analisador de imagens determinou o tamanho médio e a densidade de ocorrência das partículas, para uma ampliação de 4.000X. Com isto, somente partículas maiores que $0,1 \mu \mathrm{m}$ foram vistas.

As amostras dos aços foram, para a observação no MEV, atacadas com N ital (4\%). Com isto, deve ser observado que as dimensões dos precipitados foram superavaliadas uma vez que 0 ataque químico define não somente seus contornos, mas também a matriz corroída nas suas vizinhanças.

Já a identificação das partículas foi realizada em MET da Philips (120 kV), na Universidade Federal de São Carlos, sendo as amostras observadas com o emprego da técnica de lâminas finas.

Com o objetivo de determinar os teores de carbono e de nitrogênio em solução sólida intersticial na matriz metálica, que podem causar o envelhecimento por produzir precipitados a baixas temperaturas(1), foi realizado 0 ensaio de atrito interno. Esse ensaio foi executado nos laboratórios do Centro de $P \& D$ de Futtsu, na Nippon Steel (Japão), em equipamento ULVAC-IFM/1500, tendo sido usados corpos-de-prova distintos para a determinação do teor de cada elemento.

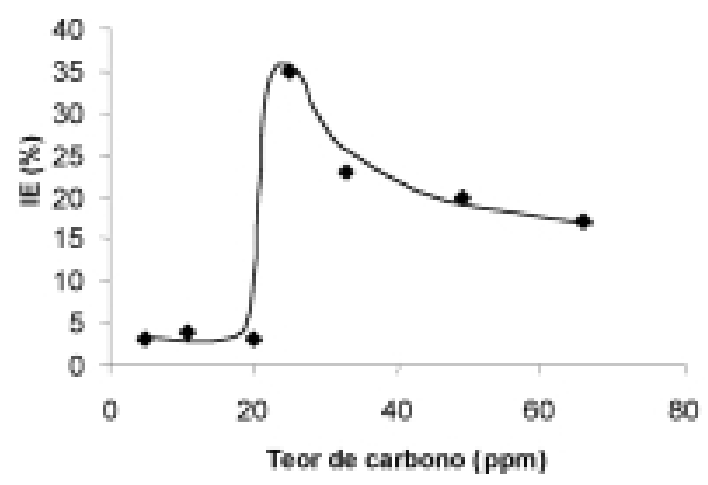

Figura 1. Aumento relativo da perda magnética (parâmetro $\mathrm{IE}$ ), em envelhecimento a $210^{\circ} \mathrm{C} / 24 \mathrm{~h}$, em função do teor residual de carbono. 


\section{RESULTADOS E DISCUSSÃO}

A Figura 2 mostra a variação do índice de envelhecimento com 0 tempo de tratamento. Observa-se que houve um aumento do IE até cerca de $24 \mathrm{~h}$. Acima desse tempo o envelhecimento ficou estabilizado.

Já a Figura 3 apresenta a evolução das componentes da perda magnética total durante 0 envelhecimento forçado. Pode ser notado que a perda histerética foi a única componente a sofrer variação, crescendo até $24 \mathrm{~h}$ de tratamento, para, a seguir, manter-se constante.

A observação em MEV da matriz metálica das amostras, nos vários tempos de envelhecimento, mostrou ter havido um aumento da quantidade de partículas de segunda fase com a extensão do tempo de tratamento. A Figura 4 mostra a matriz antes do envelhecimento e após $24 \mathrm{~h}$ de tratamento a $210^{\circ} \mathrm{C}$. Pode ser observado que o envelhecimento forçado fez aparecer uma quantidade apreciável de partículas de segunda fase maiores que $0,1 \mu \mathrm{m}$, que causam 0 envelhecimento.

0 resultado da avaliação estatística destas partículas (tamanho médio e freqüência de ocorrência), realizada em analisador de imagem, está mostrado na Figura 5. N esta figura observa-

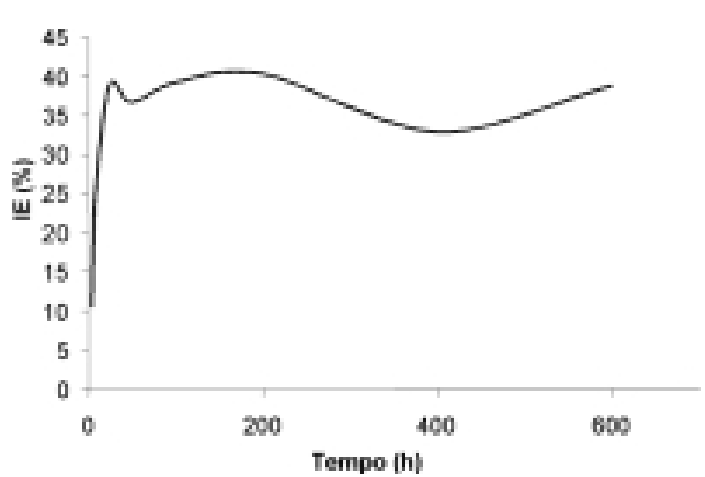

Figura 2. Evolução do índice de envelhecimento com 0 tempo para tratamentos térmicos a $210^{\circ} \mathrm{C}$.

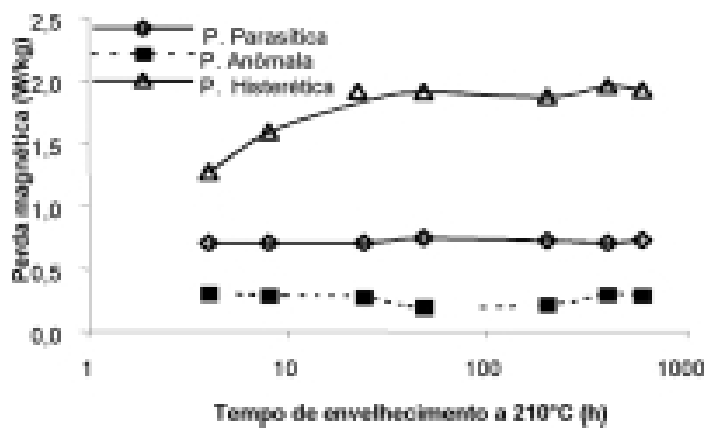

Figura 3. Variação das perdas magnéticas parciais com 0 tempo de envelhecimento forçado. se que houve um crescimento substancial do número de partículas $(>0,1 \mu \mathrm{m})$, e do seu tamanho, até cerca de $24 \mathrm{~h}$ de envelhecimento. Para tempos superiores, o tamanho médio e freqüência de ocorrência passaram a crescer muito suavemente.

As análises em MET mostraram que antes do tratamento de envelhecimento existia uma substancial quantidade de finíssimos carbonetos (dezenas de ângströns) na matriz metálica. Esses carbonetos, semicoerentes, foram identificados como LTC (low temperature carbide).

A pós envelhecimento, foram formados outros tipos de partículas, carbonetos $\varepsilon$ e cementita, de diferentes constituições cristalográficas e bem maiores, da ordem de alguns micrometros, Figura 6.

0 LTC $\mathrm{Fe}_{32} \mathrm{C}_{4}$ precipita-se normalmente a baixas temperaturas, abaixo de $100^{\circ} \mathrm{C}$, e não foi ainda seguramente identificado quanto à sua estrutura cristalina. Por seu diminuto tamanho, o carboneto LTC pouco influencia a perda histerética. $(5,6)$

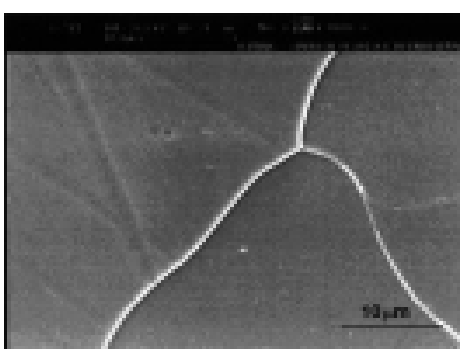

Antes do envelhecimento

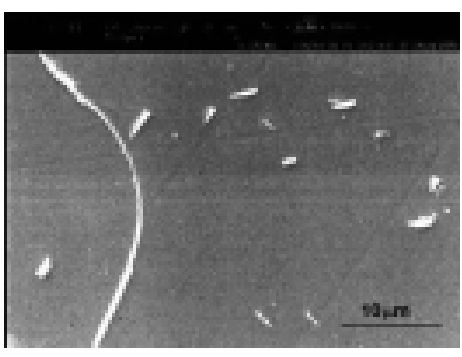

Após envelhecimento
Figura 4. Aspecto da matriz metálica antes e após envelhecimento à $210^{\circ} \mathrm{C} / 24 \mathrm{~h}$.

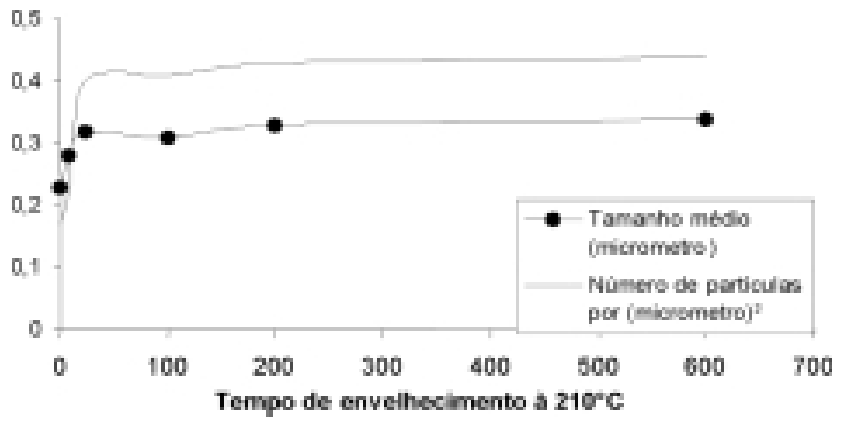

Figura 5. Evolução do tamanho médio e da quantidade de partículas de segunda fase na matriz metálica.

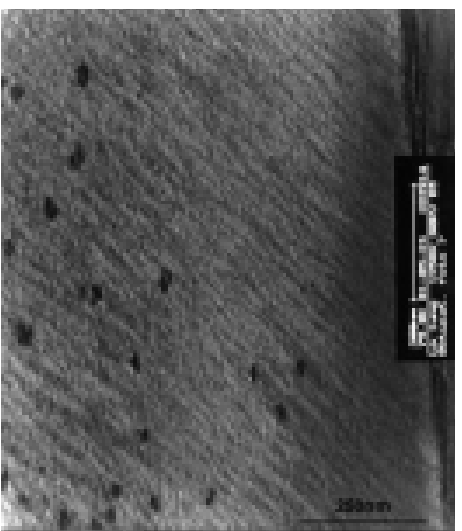

Antes do envelhecimento

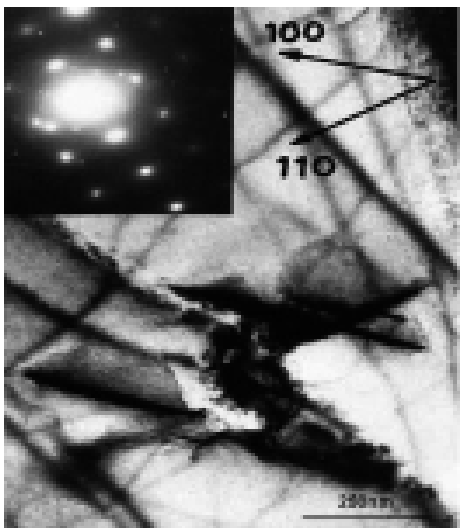

Após envelhecimento $\left(210^{\circ} \mathrm{C} / 24 \mathrm{~h}\right)$
Figura 6. Aspecto dos carbonetos precipitados na matriz metálica. 
Tabela 1. Resultados do ensaio de atrito interno (teores em \% em peso).

\begin{tabular}{lcccc}
\hline Amostra & C total & C intersticial & N total & N intersticial \\
\hline Amostra não envelhecida & 30 & 13,6 & 34 & 1,7 \\
Amostra envelhecida & 30 & 3,9 & 34 & 1,6 \\
\hline
\end{tabular}

0 carboneto $\varepsilon\left(\mathrm{Fe}_{2,4-3} \mathrm{C}\right)$, que normalmente forma-se na faixa de $100^{\circ} \mathrm{C}$ a $250^{\circ} \mathrm{C}$, possui estrutura hexagonal e plano de hábito $\{100\}$, precipitando-se na forma de discos na matriz metálica (ferrita).(6)

Já a cementita, de composição básica $\mathrm{Fe}_{3} \mathrm{C}$, apresenta estrutura ortorrômbica e plano de hábito $\{110\}$ e, normalmente, aparece como plaquetas dispostas formando dendritas, segundo a direção $<111>$ na ferrita. $(5,6)$

Deve ser observado que 0 carboneto $\varepsilon$ e a cementita, devido alcançarem tamanhos superiores a $0,1 \mu \mathrm{m}$, são efetivamente os causadores do envelhecimento magnético.

As análises por atrito interno, comparando a quantidade de carbono e de nitrogênio em solução sólida, antes e após o envelhecimento forçado à $210^{\circ} \mathrm{C} / 24 \mathrm{~h}$, Tabela 1 , indicou uma variação somente do teor de carbono. Aliás, o teor de nitrogênio em solução, nas duas condições, mostrou-se extremamente baixo. Isto certamente ocorreu em razão do relativo alto teor de Al do aço, que fixa o nitrogênio na forma de AIN .

Do exposto anteriormente, pode-se afirmar que o tratamento térmico implicou na precipitação/coalescimento de carbonetos de ferro, sendo, portanto, o carbono em solução sólida na matriz do aço o responsável pela manifestação do fenômeno do envelhecimento magnético. Com isto, é possível explicar a curva de envelhecimento da Figura 1. Assim, para teores de carbono inferiores a 20 ppm, este elemento está praticamente todo em solução sólida e há a precipitação de carbonetos muito finos, praticamente inócuos ao envelhecimento. Contrariamente, para teores acima de 50ppm, em razão de haver previamente a presença de carbonetos na matriz metálica, há 0 engrossamento dos mesmos e 0 envelhecimento torna-se tênue. No entanto, para teores entre 20 e 50 ppm de car- bono, há a geração de carbonetos (cementita e $\varepsilon$ ) em tamanho bastante efetivo para o envelhecimento.

\section{CONCLUSÕES}

A aplicação de ciclos térmicos de envelhecimento forçado, à $210^{\circ} \mathrm{C}$, nos tempos de encharque de 4 h, 8 h, 24 h, 48 h, 100 h, 200 h, 400 h e 600 h provocou, até cerca de 24 h, um substancial crescimento do índice de envelhecimento, com o mesmo praticamente ficando inalterado para maiores tempos. Foi constatado que a componente histerética da perda total ditou esse comportamento. As perdas parasítica e anômalas mostraram-se insensíveis ao envelhecimento. Isto está de acordo com informações de literatura e ocorreu em razão da perda histerética estar associada à dissipação de energia magnética pelo travamento das paredes dos domínios durante a magnetização, quando ocorre a expansão/retração ou giro dos domínios.

Além disto, foi constatado, por atrito interno e microscopia eletrônica de transmissão, que a precipitação de carbono foi responsável pelo fenômeno do envelhecimento, ou seja, carboneto $\varepsilon$ e a cementita precipitados/coalescidos durante os tratamentos de envelhecimentos passaram a interagir mais intensamente com as paredes dos domínios, causando aumento da dissipação de energia (perda magnética). Assim, por microscopia eletrônica de varredura, ficou demonstrado que a evolução do envelhecimento foi acompanhada pelo crescimento da quantidade de carbonetos maiores que $0,1 \mu \mathrm{m}$.

\section{REFERÊNCIAS}

1 LANDGRAF, F.; CAMPOS, M. F.; TEIXEIRA, J. C.; CONCILIO, G.; MURANAKA, C.; TSCHIPTSCHIN, A. WOLGIEN, V.; ZWIRMAN, N.; CORTES, C. Modelamento das perdas magnéticas de aços elétricos. In: CONGRESSO IN TERN ACION AL DE TECN O LO GIA METALÚRGICA E DE MATERIAIS DA ABM, 2., 1997, São Paulo. Anais... São Paulo : ABM, 1997.

2 SLANE, J. A.; LABU N, P. A. The relationship of magnetic properties to carbide distribution in a continuous annealed 2,3\% silicon electrical steel. In: THE AMERICAN IN ST ITUTE OF MIN IN G METALLURGICAL, AND PETRO LEUM EN GIN EERS. Energy efficient electrical steels. N ew York, 1981. p.193-204.

3 MARRA, K. M. Cinética de descarbonetação de um aço GNo semiprocessado e sua influência nas propriedades e na susceptibilidade ao envelhecimento magnético. 2002, 283p. Tese (Doutorado em Engenharia Metalúrgica) - Escola de Engenharia da Universidade Federal de Minas Gerais, Belo H orizonte, 2002.

4 BERT O TI, G. Magnetic loss modelling. IEEE Transactions on Magnetics, v. 24, n.1, p.621-630, Jan. 1988.

$5 \mathrm{ABE}, \mathrm{H}$. Carbide precipitation during ageing treatments. Scandinavian Journal of Metallurgy, v. 13, n.4, p.226239, Apr. 1984.

6 ZHU, A., N EIFE, I.; PIN K, E. Characterization of medium and low-temperature carbides in low steel by internal friction. Steel Research, v. 67, n. 11, p.507-512, N ov. 1996.

Recebido em: 11/05/2006

Aceito em: 25/10/2006

Proveniente de: CONGRESSO AN UAL DA ABM, 60., 2005, Belo Horizonte, MG. São Paulo : ABM, 2005. 